\title{
Comportamiento productivo en primer ciclo de gallinas rhode island rojas y plymouth rock barradas
}

\section{Productive behavior in the first cycle of red rhode island and plymouth rock hens, barred}

\author{
DOI: $10.46932 / \mathrm{sfjdv2n3-047}$
}

Received in: May 1st, 2021

Accepted in: Jun 30th, 2021

\author{
Adrián Hernández-Sánchez \\ Médico veterinário \\ Facultad de Medicina Veterinaria y Zootecnia, \\ E-mail: adrian021695@gmail.com \\ Ernestina Gutiérrez-Vázquez
}

Doctor en ciências

Instituto de Investigaciones Agropecuarias y Forestales,

E-mail: ernestina.gutierrez@umich.mx

Carlos Alberto Villalba-Sánchez

Maestro en ciências

Instituto de Investigaciones Agropecuarias y Forestales,

E-mail: carlos.villalba@umich.mx

\section{Rosa Elena Pérez- Sánchez}

Doctor en ciências

Facultad de Agrobiología "Presidente Juárez", Universidad Michoacana de San Nicolás de Hidalgo, km 9.5 carretera Morelia Zinapécuaro, municipio de Tarímbaro, Michoacán, México, CP 58880.

E-mail: rosa.perez@umich.mx

\section{Ruy Ortiz-Rodríguez \\ Maestro en ciências}

Facultad de Medicina Veterinaria y Zootecnia,

E-mail: ruy.ortiz@umich.mx

\section{Aureliano Juárez-Caratachea}

Doctor en ciencias

Instituto de Investigaciones Agropecuarias y Forestales,

E-mail: aurelianojuarez@ hotmail.com

\section{RESUMEN}

Se evaluó el comportamiento productivo de gallinas Rhode Island Rojas (RIR) y Plymouth Rock Barradas (PRB), durante el primer ciclo de postura, bajo las condiciones ambientales de la región del Altiplano en el estado de Michoacán, México. Para ello se utilizaron 26 gallinas doble propósito, distribuidas aleatoriamente en dos grupos: 13 RIR y 13 PRB de 20 semanas de edad, colocadas en jaulas individuales convencionales tipo batería; durante el período experimental (noviembre de 2019 a octubre de 2020), recibieron alimento comercial especial para gallinas en postura y agua, ambos ad libitum. Las variables bajo control fueron: peso vivo inicial (PVi), peso vivo final (PVf), consumo de alimento (CA), peso del 
huevo (PH), porcentaje de postura (\%P), huevos por ciclo (HPC), masa de huevo (MH), índices morfométricos (IM), índices productivos (IP). Los resultados más relevantes en los genotipos RIR y PRB fueron: PVi y PVf, 2.019 vs. $2.160 \mathrm{~kg}$ y 2.105 vs. $2.300 \mathrm{~kg}$ respectivamente $(\mathrm{P}<0.05)$; CA, RIR 124.1 vs. PRB $131.8 \mathrm{~g}(\mathrm{P}<0.05)$; $\mathrm{PH}$ y \% $\mathrm{P}$ para RIR y PRB: 59.4 vs. $61.2 \mathrm{~g}, 82.5$ vs. $64.8 \%(\mathrm{P}<0.05)$ respectivamente; HPC y MH: 287.3 vs. 226.8 y 17.217 vs. $13.721 \mathrm{~kg}$ para RIR y $\mathrm{PRB}(\mathrm{P}<0.0001)$ en su orden. En conclusión, las gallinas del genotipo Rhode Island Rojas mostraron mejor comportamiento productivo que sus contemporáneas Plymouth Rock Barradas.

Palabras clave: Gallinas semi-pesadas, Gallinas doble desempeño, Evaluación, Desempeño productivo.

\begin{abstract}
The productive behavior of Rhode Island Red (RIR) and Plymouth Rock Barradas (PRB) hens was evaluated during the first laying cycle under the environmental conditions of the Altiplano region in the state of Michoacán, Mexico. For this purpose, 26 dual-purpose hens were used, randomly distributed in two groups: 13 RIR and 13 PRB of 20 weeks of age, placed in individual conventional battery type cages; during the experimental period (November 2019 to October 2020), they received special commercial feed for laying hens and water, both ad libitum. The variables under control were: initial live weight (PVi), final live weight (PVf), feed consumption (CA), egg weight $(\mathrm{PH})$, laying percentage $(\% \mathrm{P})$, eggs per cycle (HPC), egg mass (MH), morphometric indices (IM), productive indices (IP). The most relevant results in RIR and PRB genotypes were: PVi and PVf, 2.019 vs. $2.160 \mathrm{~kg}$ and $2.105 \mathrm{vs.} 2.300 \mathrm{~kg}$ respectively (P<0.05); CA, RIR 124.1 vs. PRB 131.8 g (P<0. 05); PH and \%P for RIR and PRB: 59.4 vs. $61.2 \mathrm{~g}, 82.5$ vs. $64.8 \%(\mathrm{P}<0.05)$ respectively; HPC and MH: 287.3 vs. 226.8 and 17.217 vs. $13.721 \mathrm{~kg}$ for RIR and PRB $(\mathrm{P}<0.0001)$ in their order. In conclusion, hens of the Rhode Island Red genotype showed better productive performance than their Plymouth Rock Barradas contemporaries.
\end{abstract}

Key words: Semi-heavy hens, Double performance hens, Evaluation, Productive performance.

\title{
1 INTRODUCCIÓN
}

El crecimiento del consumo de huevo tiende a desplazar de la dieta a otro tipo de alimentos, ya que éste aporta el 4\% de las necesidades diarias de proteína (Carvajal, 2005), conteniendo aproximadamente de 6 a $7 \mathrm{~g}$ de la misma, igualmente aporta un alto contenido de aminoácidos esenciales. Además de poseer 15\% de vitamina D, 9\% de B12 y 8\% de ácido fólico (UNAM, 2007). Actualmente México se ubica como el cuarto productor de huevo a nivel mundial, después de China, EUA e India y como el principal consumidor de huevo en el mundo con $23.6 \mathrm{~kg}$ de huevo per cápita (UNA, 2018).

Según Barroeta et al. (2010) actualmente las gallinas domésticas más utilizadas para la producción de huevos son las de origen mediterráneo y las norteamericanas:

a) Las mediterráneas tienen como características principales el color blanco de su orejilla, su peso ligero y su tamaño mediano

b) Las norteamericanas o atlánticas tienden a ser grandes, son resistentes al frío, aspecto que las convierte en buenas ponedoras también en invierno, tienen la orejilla roja y su carne es de buena calidad (Oteiza, 2004). 
En relación con la raza Rhode Island Roja, Barroeta et al. (2010) mencionan que ésta toma su nombre del estado de Rhode Island (Estados Unidos de América). Surgió al cruzar las gallinas nativas que había en aquella zona en 1845 con Combatiente Malayo y Cochinchina. Es la base de las gallinas industriales actuales. Es un ave más bien grande, alcanzando el gallo un peso de 3.3 a $4 \mathrm{~kg}$ y la gallina de 2.6 a $3.3 \mathrm{~kg}$., Son resistentes a las condiciones adversas, una puesta aceptable de huevos grandes de color marrón intenso con una producción por ciclo de 211 a 225 huevos (North y Bell, 2006; Bonilla, 2018).

Por su parte Oteiza (2004) y Bonilla (2018), mencionan que la raza Plymouth Rock Barrada es una raza originaria de los Estados Unidos de América, cuya creación se remonta al año 1860. Fue importada a Europa hacia 1880. Surgió del cruce de la gallina indígena Dominicana Barrada con gallinas asiáticas como la Conchinchina y el Brahma. Es una raza grande, bastante pesada, cuyos pesos en los gallos es de 3.3 a $4 \mathrm{~kg}$ y en las gallinas de 2.6 a $3 \mathrm{~kg}$, es de porte bien derecho. Es una raza con dos fines zootécnicos claros y ambos bien desarrollados, la producción de carne y la producción de huevo, con una puesta de alrededor de 200 huevos anuales, de 55 g como mínimo.

En relación con la producción de huevo, el manejo del periodo previo al inicio de puesta que va desde las 15 semanas de edad, hasta al inicio de puesta, es determinante porque éste precisa el tamaño y número de huevos que se producirán (Barroeta et al., 2010). Por otra parte SENA (2013) menciona que la etapa de postura de las gallinas ponedoras en condiciones favorables de alojamiento, alimentación, agua, sanidad, luz y manejo adecuado, asociadas directamente a la raza y a la buena cría y desarrollo de las pollas en las etapas posteriores, da como resultado una excelente postura en cuanto a la cantidad de huevos, tamaño, calidad y eficiencia frente al consumo de alimento ya que el peso corporal y edad al inicio del período de puesta son los que definen la curva del peso del huevo para el período de postura dentro del potencial genético del ave (Barroeta et al., 2020).

Por otro lado, la FAO, (2019) señala que el tamaño del huevo no solo depende de la raza y de la obtención de una polla con las características deseables; cabe mencionar que a mayor peso logrado al término de las 18 semanas (edad madura), los huevos serán más grandes durante la vida productiva de las gallinas, en otras palabras, cuando la producción comienza antes de alcanzar el peso ideal de las pollas, menor será el tamaño del huevo. Así mismo, el tamaño puede ser influido mediante el manejo de la luz y un adecuado suministro de alimento acorde con la etapa productiva.

Ahora bien, para GMBH, (2019), la clasificación del huevo, en relación con el tamaño y el peso, producido por gallinas de la raza Lohmann Brown en su etapa productiva, son de peso menor a $63 \mathrm{~g}$ y corresponden a los porcentajes de producción más altos durante la primera mitad de la postura, y en la medida que se avanza en la etapa de producción, contrario a la anterior, se incrementan los porcentajes de huevos de mayor tamaño con pesos por encima de los $73 \mathrm{~g}$. Estos incrementos en la cantidad de huevos 
de mayor tamaño generalmente se relacionan con el aumento gradual en el peso de la ponedora. Por ello se debe intentar que el peso corporal de las pollitas esté dentro del objetivo marcado con ganancias de peso graduales, hasta la madurez sexual.

Los distintos manuales de aves de postura insisten en la importancia de lograr la uniformidad de pesos y la sincronización de la llegada a la madurez sexual entre las hembras. Esta sincronización se controla mediante los registros semanales de peso, uniformidad, conformación y consumo de las aves (Barroeta et al., 2010). Miranda (2015) refiere que la variación en el peso corporal y edad de un lote de aves, es una forma fácil y repetible de evaluar el desempeño de crecimiento de la misma, Sin embargo, aun siendo las mismas razas, en sistema de pastoreo, presentan diferentes grados de uniformidad y respuesta al comportamiento productivo (Cruz-Bermudez et al., 2021).

Así, la modelación para conocimiento, análisis e interpretación de las curvas de producción de huevos en el tiempo, es de gran interés, porque permite hacer predicciones de comportamiento, conocer el rendimiento productivo con respecto a lo esperado; además de hacer balances de parvada y análisis de los picos y meseta de postura (Agudelo et al. 2009), los cuales se abarcan desde la semana 30 a las 35 de vida, a partir de aquí se va disminuyendo la producción. «Las gallinas ponedoras, generalmente son aprovechadas por un período de 12 a 14 meses o sea desde 18 o 20 semanas de edad que inician postura, hasta las 70 o 76 que termina el primer ciclo» (Ministerio de Agricultura y Ganadería del Salvador, 2019).

Por los antecedentes ya señalados, en los que se destaca que la edad, peso y raza tienen repercusión sobre el comportamiento productivo de la parvada, es factible observar diferencia en el comportamiento de las razas semi-pesadas RIR y PRB, para los rasgos relacionados con la producción de huevo, características físicas del huevo e índices biométricos. Por ello, el objetivo del presente trabajo consistió en evaluar el comportamiento productivo de las razas Rhode Island Rojas y Plymouth Rock Barradas en un sistema semi-intensivo, bajo las condiciones ambientales de la región del Altiplano en el estado de Michoacán, México.

\section{MATERIAL Y MÉTODOS}

El estudio se desarrolló en las instalaciones avícolas de la Facultad de Medicina Veterinaria y Zootecnia de la Universidad Michoacana de San Nicolás de Hidalgo, que se localiza en el km 9.5 carretera Morelia Zinapécuaro, municipio de Tarímbaro, Michoacán, México. Durante el transcurso del año, la temperatura del municipio de Tarímbaro generalmente varía de $5{ }^{\circ} \mathrm{C}$ a $29{ }^{\circ} \mathrm{C}$ y rara vez baja a menos de $2{ }^{\circ} \mathrm{C}$ o sube a más de $32{ }^{\circ} \mathrm{C}$, su altitud es de $1860 \mathrm{msnm}$, la humedad varía del 0 al $60 \%$, el rango de precipitación es de 600-800 mm, las coordenadas del municipio son: $19^{\circ} \mathrm{C} 48^{\prime}$ de latitud Norte y 
$101{ }^{\circ} \mathrm{C} 10^{\prime} \mathrm{de}$ longitud Oeste (Ayuntamiento Tarímbaro, 2020, extraído de: https://tarimbaro.ayuntamientodigital.gob.mx/tu-municipio/medio-fisico el 16 de diciembre de 2020).

Se utilizaron 26 gallinas doble propósito, distribuidas aleatoriamente en dos grupos: 13 RIR y 13 PRB de 20 semanas de edad, previamente inmunizadas con la vacuna triple aviar: contra la enfermedad de Newcastle, coriza infecciosa y pasteurella en etapa de pre-postura (16 semanas de edad), las cuales fueron colocadas en jaulas individuales convencionales tipo batería; durante el período experimental de un año (noviembre de 2019 a octubre de 2020), recibieron alimento comercial para gallinas en postura con $16 \%$ de PC, $2850 \mathrm{kcal}$ de energía metabolizable por kg de alimento, 3.5\% de Ca, $0.5 \%$ de P disponible y agua, ambos ad libitum.

Las variables bajo control fueron: peso vivo inicial (PVi), peso vivo final (PVf), consumo de alimento (CA), peso del huevo (PH), porcentaje de huevo (\%P), huevos producidos por ciclo (HPC), masa de huevos $(\mathrm{MH})$, índices morfométricos (IM) e índices productivos (IP), diámetro ecuatorial (de) y diámetro polar de huevo (dp) e índice de forma (if). Para las mediciones de peso (kg), como las gallinas y el alimento, se utilizó una báscula digital con precisión de 5 g y para el peso del huevo, la precisión de la báscula fue de 0.1g. Para diámetro ecuatorial y polar de los huevos se utilizó un Vernier (wolfox wf9725). Para determinar el índice biométrico: índice de forma (IF) se utilizó la siguiente ecuación: IF= dt/dl x 100, como lo sugieren Juárez- Caratachea et al. (2011), donde, dt: diámetro transversal y dl: diámetro longitudinal.

La información recopilada durante el periodo experimental, se analizó mediante estadística descriptiva y, para determinar el efecto de edad y el genotipo de las gallinas sobre las distintas características productivas y aspectos físicos del huevo, se utilizó la metodología de análisis de varianza (ANOVA) y las diferencias entre genotipos se realizaron a través de la prueba de Tukey con $\alpha=0.05$ (SAS, 2010).

\section{RESULTADOS Y DISCUSIÓN}

Los resultados indican que, el PV de las aves fue afectado por el genotipo ( $\mathrm{P}=0.0019)$, no obstante, época del año $(\mathrm{P}=0.3560)$ ni la interaccion genotipo*semana $(\mathrm{P}=0.0996)$ afectaron el $\mathrm{PV}$ de las gallinas (Tabla 1), resultados que concuerdan con lo reportado por Cruz (2016), quien evaluó la ganancia de peso y PV de la semana 36 a 52 en gallinas con genotipos similares a los usados en el presente estudio, Mientras que Oteiza (2004), al evaluar aves de genotipo Rhode Island Rojas y Plymouth Rock Barradas, reporta PV de $2.8 \mathrm{~kg}$ promedio, es decir, superior al encontrado en la presente investigación: 2.019 kg para RIR y $2.160 \mathrm{~kg}$ para PRB, lo que puede atribuirse a que el PV de las gallinas evaluadas en esta investigación corresponde a gallinas jóvenes que recién inician postura, mientras que el PV reportado por Oteiza (2004) 
está referido en gallinas maduras. Asi mismo, en la tabla ya mencionada se muestra que las gallinas del genotipo PRB alcanzaron ese PV (2.160kg) a los 159.6 días de edad, momento en el cual iniciaron postura (22.8 semanas), superior a los 153.8 dias (21.9 semanas) en el que las RIR muestran un PV de 2.019kg, datos que coincide con Torres et al (2013) quienes observaron que las gallinas Rhode Island Rojas alcanzan la madurez sexual a las 22 semanas de edad en promedio.

Tabla 1. Medias de mínimos cuadrados para edad y peso vivo (PVi) al iniciar postura, así como por genotipo y época del año

\begin{tabular}{lccc}
\hline Variables/Genotipos & Rhode Island R. & Plymouth Rock B. & E.E. \\
\hline PVi promedio & $2.019^{\mathrm{a}}$ & $2.160^{\mathrm{b}}$ & 0.03 \\
Edad al i/p/,d & $153.8^{\mathrm{a}}$ & $159.6^{\mathrm{b}}$ & 0.03 \\
\hline Invierno & $2.039^{\mathrm{a}}$ & $2.152^{\mathrm{a}}$ & 0.03 \\
Primavera & $2.025^{\mathrm{a}}$ & $2.167^{\mathrm{a}}$ & 0.03 \\
Varano & $2.017^{\mathrm{a}}$ & $2.175^{\mathrm{a}}$ & 0.03 \\
Otoño & $1.997^{\mathrm{a}}$ & $2.147^{\mathrm{a}}$ & 0.03 \\
\hline
\end{tabular}

DONDE: PVI =peso vivo inicial, edad al i/p/d = Edad al iniciar postura en días, E.E.=error estândar ${ }^{\mathrm{a}, \mathrm{b}}$ Literales diferentes indican diferencia estadística $(\mathrm{P}<0.05)$ entre promedios dentro de filas.

Al evaluar la interacción genotipo*semana se observó que ésta no es significativa $(\mathrm{P}=0.0996)$ (Figura 1), no se encontró diferencia $(\mathrm{P}>0.05)$ entre genotipos en las semana de evaluación, no obstante, de acuerdo con la ecuación de regresión para el PV, en relación con la semana de evaluación se encontró que las gallinas de genotipo Plymouth Rock Barradas incrementaron $3.7 \mathrm{~g}$ semana $^{-1}$ de PV vs $1.4 \mathrm{~g}$ semana $^{-1}$ en la Rhode Island Rojas, conforme trascurrían las semanas de seguimiento (Figura 1).

Figura 1. Medias de mininos cuadrados y estimación lineal del peso corporal de las aves según genotipo y semana.
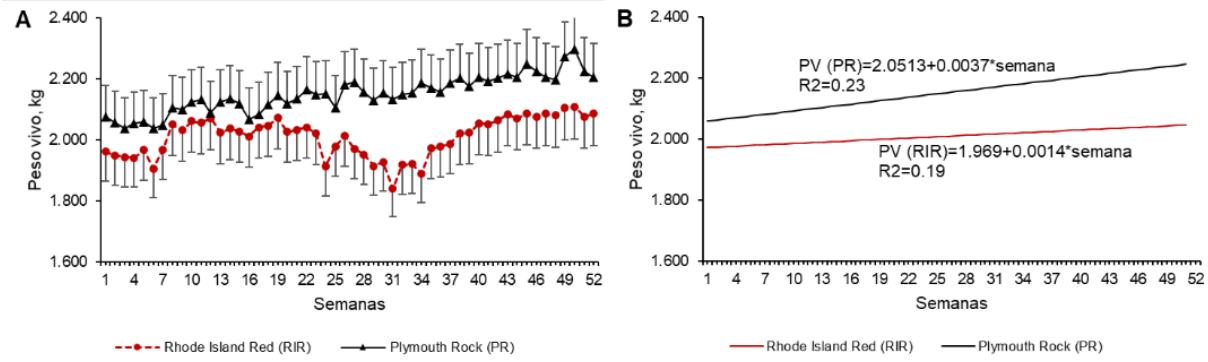

En la Figura 1 se muestra la tendencia del PVf de las gallinas, expresado a través del periodo experimental, el genotipo Plymouth Rock Barrado, a través de los mínimos cuadrados muestra un incremento permanente y sostenido durante las 52 semanas de monitoreo, mientras que el Rhode Island Rojo presenta merma de PV entre las semanas 22 a la 40 del período de postura, lapso correspondiente a las épocas de primavera y verano (Figura 2), con temperatura ambiente elevada y reducción en el consumo de alimento (Tabla 2), lo que quizá se asocie al estrés calórico, con merma en CA que repercute en el PV, como lo refieren Barroeta, (2002) y Rodríguez et al. (2011). 
En la Tabla 2 se aprecia que las gallinas de genotipo Plymouth Rock Barradas presentaron mayor $(\mathrm{P}<0.05) \mathrm{CA}$ (7.7 g más al comparar el promedio general), lo que tal vez se relaciones con la tendencia de mayor PV de este genotipo, como se muestra en la Figura 1. De acuerdo con Calvo (2015) las aves Plymouth Rock Barradas se originaron por una cruza entre gallinas indígenas Dominicas x Conchinchinas x Brhaman, consideradas las dos últimas como razas pesadas de las más grandes, lo que tal vez explica el mayor PV y mayor CA.

Tabla 2. Medias de mínimos cuadrados para consumo de alimento (CA), según genotipo y época del año

\begin{tabular}{llll}
\hline & \multicolumn{3}{c}{ Consumo de alimento (g/día) } \\
\hline Época del año & Rhode Island R. & Plymouth Rock B. & E.E. \\
\hline Promedio general & $124.1^{\mathrm{a}}$ & $131.8^{\mathrm{b}}$ & 0.001 \\
Primavera & $107.6^{\mathrm{a} 1}$ & $119.5^{\mathrm{b} 1}$ & 0.001 \\
Verano & $128.1^{\mathrm{a} 2}$ & $132.6^{\mathrm{a} 2}$ & 0.001 \\
Otoño & $132.8^{\mathrm{a} 2}$ & $146.9^{\mathrm{b} 2}$ & 0.001 \\
Invierno & $129.7^{\mathrm{a} 3}$ & $133.4^{\mathrm{b} 3}$ & 0.001 \\
\hline
\end{tabular}

${ }^{\mathrm{a}, \mathrm{b}}$ Literales diferentes indican diferencia estadística $(\mathrm{P}<0.05)$ dentro de columnas.

$1,2,3$ Numerales diferentes indican diferencia estadística $(\mathrm{P}<0.05)$ dentro de las filas.

En relación con el efecto de la época del año sobre los indicadores productivos PV y CA (Tablas 1 y 2), ésta no afectó el comportamiento del PV (>0.05) de los genotipos en cuestión, pero si el CA $(\mathrm{P}<0.001)$. Al respecto, Merino (2005) señala que, la temperatura y la humedad de confort dentro de la caseta de gallinas ponedoras son de 18 a $24^{\circ} \mathrm{C}$ y de 60 a $65 \%$ respectivamente. Por su parte el Centro Meteorológico Nacional reporta para el área de influencia donde se estableció la etapa experimental, una temperatura promedio de $29.0{ }^{\circ} \mathrm{C}$ y una humedad relativa de $65.4 \%$ durante las épocas de primavera, otoño e invierno (CNA, 2019).

De acuerdo con Merino (2005), el estrés calórico inicia a partir de $\operatorname{los} 26^{\circ} \mathrm{C}$, pero es aparente hasta los $29^{\circ} \mathrm{C}$, cuando inicia el jadeo. Está demostrado que las variables ambientales como alta temperatura y humedad relativa, afectan directamente en balance energético, metabólico y comportamiento productivo de las ponedoras, comprometiendo significativamente la temperatura corporal y el producto final; el huevo (Barroeta, 2002 y Rodríguez et al., 2011), lo que quizá explique porqué en la época de primavera, ambos genotipos presentaron el más bajo CA.

Con respecto al índice de producción, el genotipo Rhode Island Rojo presentó un \%P superior (78.6\%) con respecto al \% P del genotipo PRB fue de 61.9\% ( $<<0.05)$ (Figura 2) Este mismo genotipo RIR, en la época de invierno registra un \%P de 91.5, lo que equivale al pico de postura (semanas 30 a 36), resultados que coinciden con los valores señalados por Oteiza (2004), dicho autor establece que las gallinas RIR producen de 280 a 320 huevos por año, lo que traducido en \%P equivale al dato antes indicado: 76.7 a $87.7 \%$, respectivamente. 
Figura 2. Medias de mininos cuadrados para el porcentaje de postura de las aves de acuerdo con el genotipo, semana y época.

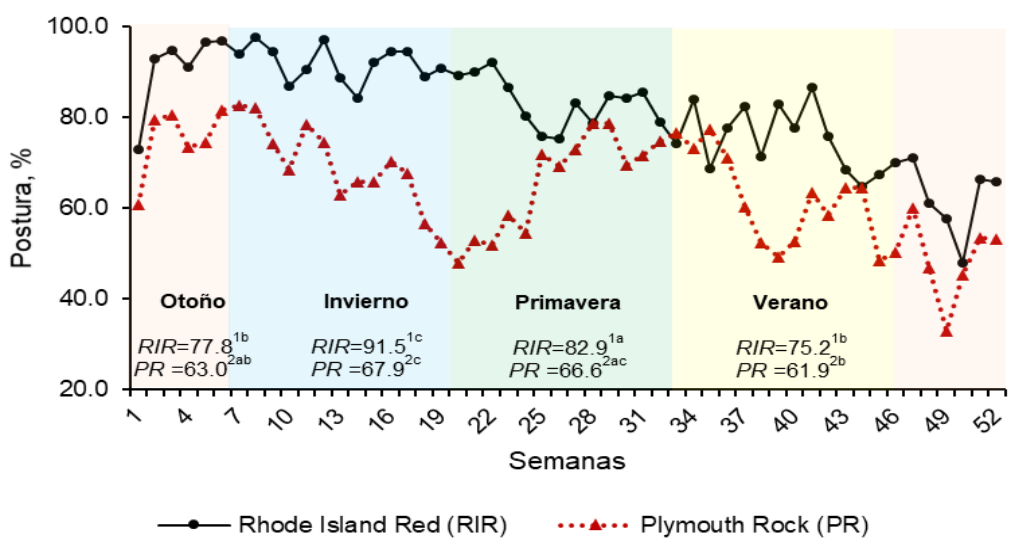

${ }^{a, b, c}$ Literales diferentes indican diferencia estadística $(\mathrm{P}<0.05)$ entre época y dentro de cada genotipo. ${ }^{1,2}$ Numerales diferentes indican diferencia estadística $(\mathrm{P}<0.05)$ dentro de época y entre genotipos.

El \%P del genotipo PRB es inferior (16.7\%), al genotipo RIR como se observa en la Gráfica 2, sin embargo, el \%P aquí observado es aun superior al que resulta, según los 200 a 220 huevos por gallina al año que atribuye Morfín (2007) a este genotipo. No obstante que ambos genotipos mostraron un \%P con altibajos y que las aves PRB mostraron menor \%P, se debe destacar que, de la semana 25 a la 35, las PRB mostraron su mayor \% $\mathrm{P}$, justamente durante las épocas de primavera y verano, cuando la temperatura ambiente superó los $30^{\circ} \mathrm{C}$ (Tabla 3), esto tal vez guarda relación con rusticidad, adaptabilidad y resistencia cambios ambientales de este genotipo, como la indican Conet et al. (2014). Finalmente, en ambos grupos genéticos el \%P inicia descenso después de la semana 40 de postura.

Figura 3. Medias de mininos cuadrados para el peso del huevo de las aves de acuerdo con el genotipo, semana y época.

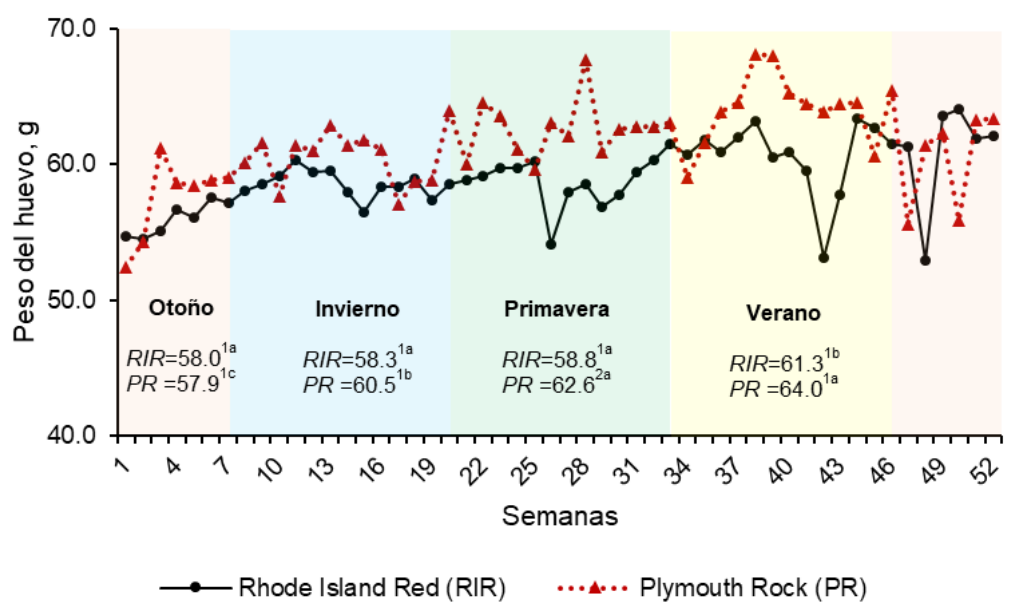

${ }^{a, b, c}$ Literales diferentes indican diferencia estadística $(\mathrm{P}<0.05)$ entre época y dentro de cada genotipo. ${ }^{1,2}$ Numerales diferentes indican diferencia estadística $(\mathrm{P}<0.05)$ dentro de época y entre genotipos.

Tabla 4. Datos del Observatorio Meteorológico de Morelia, Michoacán, Año 2019 (Comisión Nacional del Agua, 2019)

\begin{tabular}{|l|l|l|l|l|l|l|l|l|l|l|l|l|}
\hline $\begin{array}{l}\text { Elemento } \\
\text { Observado }\end{array}$ & Ene & Feb & Mar & Abr & May & Jun & Jul & Ago & Sep & Oct & Nov & Dic \\
\hline $\begin{array}{l}\text { T. P. M. } \\
{ }^{\circ} \mathrm{C}\end{array}$ & 25.1 & 27.2 & 31.7 & 32.3 & 32.1 & 30.9 & 30.3 & 29.9 & 29.8 & 26.1 & 23.4 & 22.4 \\
\hline
\end{tabular}




\begin{tabular}{|l|l|l|l|l|l|l|l|l|l|l|l|l|}
\hline H. R. P. \% & 67 & 68 & 54 & 55 & 69 & 74 & 76 & 78 & 78 & 66 & 62 & 64 \\
\hline
\end{tabular}

DONDE: T.P.M. = temperatura promedio máxima y H.R.P. = humedad relativa promedio.

El indicador productivo $\mathrm{PH}$, muestra un comportamiento similar en ambos genotipos (Figura 3), la tendencia es a incrementar conforme transcurren las semanas de observación. También se evidencia que el genotipo PRB produce huevos de mayor peso, de modo que la diferencia entre las variables PVi y PVf parecen estar relacionadas con el PH al iniciar y terminar la postura. En relación con estos hallazgos García et al. (2016), observaron que el peso corporal y la edad influyen directa y positivamente sobre los indicadores bioproductivos de las gallinas, entre ellos el huevo.

Los resultados de la Tabla 5 muestran que, los valores de diámetro polar o longitudinal, diámetro ecuatorial o transversal, índice de forma y peso del huevo no presentan diferencias estadísticas (P>0.05) por efecto del genotipo. Sin embargo, las variables huevos por ciclo de producción y masa de huevos, muestran efectos altamente significativos del genotipo ( $\mathrm{P}<0.0001)$, en favor de las aves RIR.

Tabla 5. Comparación de índices morfométricas y productivos entre los genotipos Rhode Island Rojo (RIR) y Plymouth Rock Barrado PRB)

\begin{tabular}{|c|c|c|c|c|c|c|}
\hline Variable & Genotipo & Media \pm D.E & C. de V. & V mín & $\mathrm{V}$ máx & $\mathrm{V}$ de $\mathrm{P}$ \\
\hline d. p., mm & RIR PRB & $\begin{array}{l}56.1 \pm 2.155 .7 \pm \\
2.5\end{array}$ & $\begin{array}{l}3.73 \\
4.49 \\
\end{array}$ & $\begin{array}{l}51.4 \\
49.3\end{array}$ & $\begin{array}{l}59.8 \\
58.7\end{array}$ & 0.6964 \\
\hline d. e., $\mathrm{mm}$ & RIR PRB & $\begin{array}{l}42.7 \pm 1.442 .2 \pm \\
1.5\end{array}$ & $\begin{array}{l}3.34 \\
3.78\end{array}$ & $\begin{array}{l}39.6 \\
39.2\end{array}$ & $\begin{array}{l}44.6 \\
44.6\end{array}$ & 0.4666 \\
\hline $\begin{array}{l}\text { Índice de } \\
\text { forma, \% }\end{array}$ & RIR PRB & $\begin{array}{l}76.2 \pm 3.575 .8 \pm \\
1.9\end{array}$ & $\begin{array}{l}4.63 \\
2.61\end{array}$ & $\begin{array}{l}67.5 \\
72.1\end{array}$ & $\begin{array}{l}81.1 \\
79.4\end{array}$ & 0.7643 \\
\hline $\begin{array}{l}\text { Peso del } \\
\text { huevo, g }\end{array}$ & RIR PRB & $\begin{array}{l}59.9 \pm 3.360 .4 \pm \\
4.3\end{array}$ & $\begin{array}{l}5.46 \\
7.08\end{array}$ & $\begin{array}{l}53.1 \\
50.1\end{array}$ & $\begin{array}{l}65.2 \\
66.7\end{array}$ & 0.6922 \\
\hline $\begin{array}{l}\text { Huevos/ ciclo, } \\
\text { u }\end{array}$ & RIR PRB & $\begin{array}{l}287.3 \pm 29.1 \\
226.8 \pm 36.9\end{array}$ & $\begin{array}{l}10.1 \\
16.2\end{array}$ & $\begin{array}{l}230.0 \\
163.0\end{array}$ & $\begin{array}{l}320.0 \\
300.0\end{array}$ & 0.0001 \\
\hline $\begin{array}{l}\text { Masa de } \\
\text { huevos, kg }\end{array}$ & RIR PRB & $\begin{array}{l}17.217 \pm 2.25 \\
13.721 \pm 2.57\end{array}$ & $\begin{array}{l}13.1 \\
18.7\end{array}$ & $\begin{array}{l}13.054 \\
10.214\end{array}$ & $\begin{array}{l}19.597 \\
18.479\end{array}$ & 0.0001 \\
\hline
\end{tabular}

Donde: D.E. = desviación estándar, C. de V. = coeficiente de variación, V min = valor mínimo, V máx = valor máximo, V de $\mathrm{P}=$ valor de $\mathrm{P}$, d.p. = diámetro polar y d.e. = diámetro ecuatorial.

En relación con el número promedio de huevos producidos durante el primer ciclo de postura por genotipo: $287.3 \pm 29.1$ unidades para RIR y $226.8 \pm 36.9$ unidades para PRB, con una diferencia $(\mathrm{P}<0.0001)$ de 60.5 huevos en favor de las gallinas RIR, lo que coincide con los datos reportados por Oteiza (2004), quien señala una producción de 280 a 320 huevos por gallina en un año para la RIR y Morfin (2007), 200 a 220 huevos/gallina/año para las PRB. Consecuentemente, el número de huevos y el peso de los mismos determinan los kg o masa de huevo generada por los genotipos aquí evaluados en el primer ciclo de postura.

Tabla 6. Estimación de costos de producción por concepto de alimentación y eficiencia alimentaria entre los genotipos RIR y PRB 


\begin{tabular}{|l|l|l|l|l|l|}
\hline FENOTIPO & $\mathrm{CA} / \mathrm{kg} / \mathrm{c}$ & $\mathrm{kg} / \mathrm{h} / \mathrm{g} / \mathrm{c}$ & $\begin{array}{l}\text { Precio del } \\
\text { alimento, } \$\end{array}$ & $\begin{array}{l}\text { Conversión } \\
\text { alim/kg:kg }\end{array}$ & $\begin{array}{l}\text { C de } \mathrm{p} / \mathrm{c} \text { de } \\
\text { alimentación }\end{array}$ \\
\hline RIR & 45.296 & 17.217 & $7.50 \$$ & $2.631: 1$ & $339.72 \$$ \\
\hline PRB & 48.107 & 13.741 & $7.50 \$$ & $3.506: 1$ & $360.80 \$$ \\
\hline $\begin{array}{l}\text { Diferencias entre } \\
\text { genotipos }\end{array}$ & 2.811 & 3.496 & & 0.875 & $21.08 \$$ \\
\hline
\end{tabular}

Donde: RIR: Rhode Island Rojas, PRB: Plymouth Rock Barradas, CA/g/c:: consumo de alimento por gallina y por ciclo, $\mathrm{kg} / \mathrm{h} / \mathrm{g} / \mathrm{c}$ : $\mathrm{kg}$ de huevos por gallina y por ciclo, $\mathrm{C}$ de p/c de alimentación: costos de producción por concepto de alimentación.

El análisis de los costos de producción por concepto de alimento en ambos genotipos evidencia que, son más eficientes y más rentables las aves RIR toda vez que las diferencias entre ambos grupos las favorecen. En la Tabla 6 se muestra que la diferencia en CA por gallina por ciclo $(2.811 \mathrm{~kg})$, en los $\mathrm{kg}$ de huevo producidos por gallina por ciclo (3.496), en la conversión de alimento a kg de huevo (0.875) y en el costo de producción por concepto de alimento (21.08) respaldan el comportamiento productivo de las gallinas RIR, en comparación con las PRB, bajo las condiciones ambientales propias de la región del Altiplano en Michoacán, Máxico. Lo anterior coincide con los hallazgos de Cruz (2016) quien también encontró diferencia significativa ( $\mathrm{P}<0.05)$, en favor de la raza RIR, la cual superó en un $1.21 \%$ a la raza PRB (13,220 g/ave vs 13,060 g/ave, respectivamente). El precio del alimento con base al cual se realizó el presente análisis, corresponde al precio del kg de alimento en el mercado en pesos mexicanos, al momento de llevar a cabo la investigación (noviembre de 2019 a octubre de 2020).

\section{CONCLUSIONES}

El peso vivo inicial, el peso vivo final y el consumo de alimento fueron superiores en las gallinas del genotipo Plymouth Rock Barradas, en comparación con sus contemporáneas Rhode Island Rojas.

El número de huevos producidos por ciclo de puesta, el porcentaje de huevos y la masa de huevos, resultaron significativamente superiores en las gallinas Rhode Island Rojas en comparación con las Plymouth Rock Barradas.

El consumo de alimento por genotipo por ciclo, los kgs de huevo por gallina por ciclo, la conversión de alimento en huevo y los costos de producción por concepto de alimento, resultaron mejores en las aves Rhode Island Rojas.

\section{AGRADECIMIENTO}

Los autores agradecen a la Coordinación de la Investigación Científica de la Universidad Michoacana de San Nicolás de Hidalgo, el financiamiento otorgado para la presente investigación. 


\section{REFERENCIAS BIBLIOGRÁFICAS}

Agudelo, D. A; Cerón, M. F. Y Restrepo, L. F. 2009. Modelación de las funciones de crecimiento aplicadas a la producción animal. Revista Colombiana de Ciencias Pecuarias 21 (1):39-58.

Barroeta, A. 2002. Formación del huevo. En: Lecciones sobre huevo. $1^{\text {a }}$ Edición, Madrid, España, Instituto del Huevo. pp. 45-56

Barroeta, A. C., Izquierdo, D., \& Pérez, J. F. Manual de avicultura .2010. [En línea]. Consultado el 11 de enero de 2019 , https://previa.uclm.es/profesorado/produccionanimal/ProduccionAnimalIII/GUIA\%20AVICULTURA_ castella.pdf.

Barroeta, A.C., Verge, G., Ciria, N. 2020. Alimentación de la gallina ponedora. Servicio de Nutrición y Bienestar (SNIBA). Departamento de Ciencia Animal de los Alimentos - UAB, Balterra, Barcelona, España.

Bonilla, F.S.S. 2018. Parámetros productivos y reproductivos en cuatro razas de gallinas (Gallus gallus domesticus) de doble propósito. Central Amercan Journals Online, vol 9(9):1-10.

Calvo, F.C. 2015. Principales razas de gallinas. En: Producción Animal III: Genética Avícola, Especialidad a Distancia. Universidad Nacional Autónoma de México.

Carvajal, A. 2005. Hábitos de consumo de huevos, calidad nutricional y relación con la salud. Departamento de Nutrición. Facultad de Farmacia, Universidad Complutense de Madrid. http://institutohuevo.com/scripts/docs/47/465.pdf.

Comisión Nacional del Agua, (CONAGUA) 2019. Datos del Observatorio Metereológico de Morelia, Michoacán, México.

Conet, Z., Advínculos, S., Fernández, F., Martínez, A., Librera, J., Dottavio, A., Di Masso, P. 2014. Caracteres productivos a la madurez sexual y peso del huevo en función de la edad de postura de tres grupos genéticos de gallinas camperas. Compendio de Ciencias Veterinarias. 04(01): 7-12.

Cruz, S. A. 2016. Caracterización del comportamiento productivo de dos razas de gallinas ponedoras durante las semanas 36 a 52. (Tesis de licenciatura). División de Ciencia Animal, Departamento de Nutrición. Universidad Autónoma Agraria Antonio Narro, Saltillo, Coahuila, México.

Cruz-Bermudez, A., WingChing-Jones, R., Zamora-Sánchez, R. 2021. Gallinas sex Link Negro y Rhode Island Red con acceso a pastoreo: productividad y comportamiento. Agronomía Mesoamericana, vol. 32(2):599-618.

FAO, 2019. Alojamiento y manejo de las aves de corral. [En línea] Consultado el 27 de enero de 2019, http://www.fao.org/3/a-al734s.pdf..

García, D., Colas, M., López, W., Pérez, E., Sánchez. P, A., Lamazares. P, M., \& Grandía, R. 2016. El peso corporal y su efecto sobre indicadores bioproductivos en gallinas White Leghorn L33. Revista de la Facultad de Medicina Veterinaria y de Zootecnia, 63(3): 188-200. 
GMBH. 2019. Ponedoras. Guía de manejo, Lohmann Brown-Classic. [En línea] Consultado el 27 de febrero de 2019, http://ibertec.es/docs/productos/lbcbrown.pdf.

http://es.weatherspark.com 2019. Clima promedio en Morelia, Michoacán, México durante el año, (Consultado el 16 de diciembre de 2020).

Juárez-Caratachea, A., Gutiérrez-Vázquez, E., Pérez-Sánchez, R.E., Román-Bravo, R.M., \& OrtizRodríguez, R. 2011. Evaluación física de la calidad externa e interna del huevo de pavas nativas (Melleagris gallipavo g.). Revista Científica, 21(6):524-532.

Merino, G.R. 2005. Ventilación en las casetas avícolas. División del Sistema de Universidad Abierta y Evolución a Distancia. Universidad Nacional Autónoma de México. D.F.pp.41-53.

Ministerio de Agricultura y Ganadería del Salvador. 2019. Guía para el manejo de gallinas ponedoras. [En línea]. Consultado el 03 de Septiembre de 2018, www.transparencia.gob.sv/institutions/mag/documents/119835/download.

Miranda G. S., 2015. Uniformidad en Gallinas Reproductoras Pesadas. [En línea] Consultado el 27 de julio de 2018, https://www.engormix.com/avicultura/articulos/uniformidad-gallinas-reproductoraspesadas-t32254.htm.

Morfin, L.L. 2007. Manual de Producción de gallinas ponedoras de postura. Universidad Nacional Autónoma de México.

North, M. O. \& Bell, D. D., .2006. Manual de producción avícola. Tercera ed. México: El Manual Moderno, S.A. de C.V.

Oteiza, F. J, .2004. Razas de gallinas: origen y descripción. Primera ed. Editorial Trillas, México, pp.165

Rodríguez, A., López, E.,Valdez, Y. 2011. Evaluación de indicadores agregados ambientales en la producción de huevo. OIDLES, vol. 5(10):1-30.

SAS (Statistical Analysis System). 2010. SASTM Software Version 6.0. Statistical Analysis System Institute. USA.

Servicio Nacional de Aprendizaje, (SENA) 2013. Manual de gallinas ponedoras. [En línea] Consultado el 16 de febrero de 2019, https://es.slideshare.net/jaimeaugusto/manual-de-gallina-ponedora-sena.

Torres, A., Bañuelos, V,R., Meza, L, C., Rodríguez, F, H., Ramón, R., \& Echavarría, C, F. G., 2013. Indicadores Productivos de Gallinas Rhode Island en un Sistema de Traspatio en la Localidad de Valle de San Francisco, Loreto, Zacatecas. [En línea] Consultado el 07 de junio de 2019, http://congresos.cio.mx/memorias_congreso_mujer/archivos/extensos/sesion3/S3-BCA13.pdf

UNA, 2018. SITUACIÓN DE LA AVICULTURA MEXICANA [En línea] Consultado el 02 de septiembre de 2018, http://www.una.org.mx/index.php/panorama/situacion-de-la-avicultura-mexicana

UNAM. 2007. El huevo. [En línea] Consultado el 19 de agosto de 2018, http://avalon.cuautitlan2.unam.mx/pollos/m2_9.pdf. 